\title{
Brain activation in patients with congenital bilateral hearing impairment
}

\author{
Juen-Haur Hwang ${ }^{\mathrm{a}, \mathrm{d}}$, Chang-Wei Wub ${ }^{\mathrm{b}}$, Chia-Wei Lee ${ }^{\mathrm{b}}$, Jyh-Horng Chen ${ }^{\mathrm{b}}$ and \\ Tien-Chen Liu ${ }^{c}$
}

\begin{abstract}
${ }^{a}$ College of Medicine, Graduate Institute of Clinical Medicine, ${ }^{b}$ Interdisciplinary MRI/MRS Lab, Department of Electrical Engineering, 'Department of Otolaryngology, National Taiwan University Hospital, Taipei and ${ }^{\mathrm{d} D e p a r t m e n t ~ o f ~ O t o l a r y n g o l o g y, ~ B u d d h i s t ~ D a l i n ~ T z u ~ C h i ~}$ General Hospital, Chiayi, Taiwan
\end{abstract}

Correspondence to DrTien-Chen Liu, Department of Otolaryngology, National Taiwan University Hospital, Taipei, Taiwan Tel: +8862 23123456, ext 5896; fax: +8862 23410905; e-mail: liuent@ntu.edu.tw

Received I8 May 2007; accepted 20 May 2007

Twelve patients with idiopathic, congenital, symmetric, moderateto-severe sensorineural hearing loss participated in this study. Functional magnetic resonance imaging was performed while speech sounds were presented to each patient monaurally. Notable blood oxygenation level-dependent responses were clustered mainly in the superior temporal gyrus and transverse temporal gyrus of both hemispheres during right and left ear stimulation.
In addition, the middle temporal gyrus of the right hemisphere was activated during right ear stimulation. The activation pattern was very similar to that of participants with normal hearing. Thus, as long as peripheral acoustic stimulation has not been totally absent from childhood, the classical activation pattern can be elicited in patients with congenital bilateral hearing impairment. NeuroReport 18:1483-1486 (C) 2007 Lippincott Williams \& Wilkins.

Keywords: brain activation, congenital hearing impairment, functional magnetic resonance imaging

\section{Introduction}

The pattern of auditory cortical activation changes when the inputs to the auditory system change during development. Anatomically, deaf and hearing patients did not differ in total volume or in the grey matter volume of Heschl's gyrus. The grey/white matter ratio in the bilateral Heschl's gyrus and superior temporal gyrus (STG) was, however, larger in deaf patients than in hearing participants. This pattern was, however, not observed for the temporal lobe as a whole [1]. Functionally, decreased cerebral glucose metabolism in the auditory-associated cortices was observed in younger patients with prelingual deafness. As the patients aged, the glucose metabolism returned to the normal level or even exceeded it owing to the expansion of the afferent neural network through the establishment of new connections with other sensory systems [2]. In addition, postlingual deaf patients were found to have a significantly lower glucose metabolism in both the anterior cingulate gyri and the superior temporal cortices, and in the right parahippocampal gyrus [3].

In the past, most functional imaging studies on the activation patterns used patients with total deafness or unilateral deafness and seldom those with congenital, bilateral, moderate-to-severe hearing impairment. The latter individuals do not receive appropriate auditory stimulation during development and therefore have poorer speech-discrimination scores in behavioral studies [4]. As little is known about brain activation in these patients, we used functional magnetic resonance imaging (fMRI) to investigate their activation patterns during speech perception.

\section{Methods}

Patients

Twelve healthy adults (six men and six women; age range: 30-53 years, mean age: 40.5 years) with bilateral symmetric, moderate-to-severe sensorineural hearing loss of idiopathic etiology participated in this study. Their audiograms were all of the downward-sloping type. The mean pure tone average was $58 \mathrm{~dB}$ HL for the right ear and $59 \mathrm{~dB}$ HL for the left ear. The mean word-discrimination score was significantly lower in the hearing-impaired group $(82.6 \%$ for the right ear and $83.0 \%$ for the left ear) than in the normalhearing group $(97.3 \%$ for the right ear and $98 \%$ for the left ear) (Student's $t$-test, $P<0.001)$. None of these patients had any experience using hearing aids or sign language. Twelve age-matched and sex-matched, normal-hearing healthy participants served as controls.

Experimental design and acoustic stimulation Using fMRI, we examined brain activation in response to a passive listening of spoken text. A prerecorded, continuously speaking, male voice reading Chinese sentences from a newspaper article describing social topics at a speed of 90 words/min was used as the speech stimulus. Articles containing factual information were selected and those containing emotional descriptions or opinions were excluded. The intensity of the stimulus (loudness of the speech signal) was adjusted to a level that was comfortable for each patient. The speech sounds were delivered to each patient monaurally. The order of ear stimulation was randomized and counterbalanced across participants. 
Each experimental series consisted of four 40-s sessions of 'baseline' (OFF) alternating with 'activation' (ON), during which prepared auditory stimuli were delivered. The script was divided into four segments, which were delivered during the four 'activation' (ON) periods without repetition. The patients were instructed to listen attentively to the spoken text and were not given any linguistic task. To monitor their levels of attention during the experiment, participants were asked to press a button gently at the end of each sentence. The number of hits and total number of sentences read were counted during scanning. After the experiment, patients in both groups achieved similar alertness and attention levels regardless of the ear stimulated.

\section{Functional magnetic resonance imaging scanning and data analysis}

Functional measurements were performed using a 3-T machine (MedSpec; Bruker, Ettlingen, Germany) with a quadrature transmit-receive head coil. Functional images were generated by means of $\mathrm{T}_{2}{ }^{*}$-weighted gradient-echo echo-planar imaging (EPI) with a $90^{\circ}$ Hermite-shaped radiofrequency pulse. Ten 6-mm sections, aligned with the anterior-posterior commissural line, were acquired to cover the entire auditory cortex. Imaging parameters were field of view $=30 \times 30 \mathrm{~cm}^{2}$, matrix $=128 \times 128$, repetition time$8000 \mathrm{~ms}$, and echo time $=40 \mathrm{~ms}$. To reduce possible masking effects of scanner noise on the experimental stimuli, a repetition time of $8 \mathrm{~s}$ was adopted [5]. The time of EPI acquisition was set at the first $2000 \mathrm{~ms}$, and the beginning of each block was synchronized with the acquisition of the first slice of each brain volume. Stimulus presentation was triggered by the EPI sequence to start $200 \mathrm{~ms}$ after the previous scan and to fall within the interscan gap of $6 \mathrm{~s}$. Five images/slice were obtained in each block and 40 images/ slice in one series.

Functional data were analyzed with the Statistical Parametric Mapping software version 2 (SPM2; Wellcome Department of Cognitive Neurology, London, UK), and a software program was written by the authors using MATLAB 7.0.1 (MathWorks Inc., Natick, Massachusetts, USA). The data were realigned with the first image of each session to remove image drift using a six-parameter rigid body model. The images were spatially normalized to the Montreal Neurological Institute template. The three-dimensional datasets (scans) were resampled to $2 \mathrm{~mm}^{3}$ isotropic voxels. The normalized images were spatially smoothed to compensate for inter-patient variation in anatomy and potential normalization error using Gaussian kernels of $6 \mathrm{~mm}$ (for a fixed-effect model analysis) and $8 \mathrm{~mm}$ (for a random-effect model analysis). A high-pass filter was applied at the cutoff point of $128 \mathrm{~s}$. Finally, coordinates in Montreal Neurological Institute space were adjusted to the Talairach coordinate system to confirm the location of activation. Statistically, a boxcar function was applied as a reference function. Individual analysis for each patient was performed using a fixed-effect model with a height threshold of $P<0.001$ (corrected, $T=4.93$ ) and an extent threshold of $P=0.008(K=20)$. For the group study, a random-effect model analysis was performed. The images were globally normalized to a mean of 100 , and a one-sample $t$-test was performed on the average images to create an SPM map with a height threshold of $P<0.001(T=4.93)$ and an extent threshold of $P=0.008 \quad(K=20)$. In this study, we were interested in the activation of the association areas of the auditory cortex. Also, the activation signals were clustered in the bilateral temporal lobes of both groups. We, therefore, selected two cuboidal regions of interest $(100 \times 80 \times 16 \mathrm{~mm})$ centered on the Talairach coordinates $[(x=46, y=24, z=3)$ for the right side and $(-46,24,3)$ for the left side] to cover the Heschl's, superior temporal, middle temporal, and angular gyri of both hemispheres.

The average blood oxygenation level-dependent (BOLD) signal intensity changes were determined for each voxel. Between-group differences in signal intensity in the auditory cortex under different stimulation conditions were then compared using the Student's $t$-test, in which $P<0.05$ indicated a significant difference.

\section{Results}

\section{Normal-hearing patients}

By group analysis, we found that voxels with positive BOLD signals were mainly clustered in the STG [Brodmann areas (BAs) 22 and 29] and the transverse temporal gyrus (BA 41) of both hemispheres. Bilateral midbrain and left thalamus were also activated by right ear stimulation. In addition, the right insula (BA 13), bilateral thalamus, right posterior cingulate gyrus (BA 29), and middle temporal gyrus (BA 19) were also activated by left ear stimulation (Fig. 1 and Table 1).

The mean signal intensities for the right and left hemispheres were 0.37 (SD, 0.35) and $0.73(\mathrm{SD}, 0.38)$, respectively, during right ear stimulation; and $0.83(\mathrm{SD}, 0.88)$ and 0.93 (SD, 0.30), respectively, during left ear stimulation. The signal changes were prominent in the hemisphere contralateral to the stimulated right ear (Student's $t$-test, $P=0.016$ ), but not during left ear stimulation $(P=0.760)$.

\section{Hearing-impaired patients}

Voxels with positive BOLD signals were mainly clustered in the STG (BA 22) and transverse temporal gyrus (BA 41) of both hemispheres. In addition, the middle temporal gyrus (BA 21) of the right hemisphere was activated during right ear stimulation (Fig. 1 and Table 2).

The mean signal intensities for the right and left hemispheres were 0.89 (SD, 0.58) and 1.17 (SD, 0.43), respectively, during right ear stimulation and 1.05 (SD, 0.47) and 0.92 (SD, 0.34), respectively, during left ear stimulation. The signal changes seemed to be more prominent in the hemisphere contralateral to the stimulated right $(P=0.074)$ or left ear $(P=0.048)$.

\section{Normal-hearing versus hearing-impaired participants}

The activation patterns in both hearing-impaired and normal-hearing participants were quite similar. The mean group activation maps, however, showed that the apparent extent of activation was slightly greater in hearing-impaired patients during left or right ear stimulation (Fig. 1). In addition, compared with normal-hearing participants, the hearing-impaired patients had signal intensities that were slightly increased in both hemispheres during right ear stimulation (Student's $t$-test; $P=0.009$ for the right hemisphere; $P=0.0125$ for the left hemisphere). The differences were, however not statistically significant during left ear stimulation (Student's $t$-test; $P=0.4375$ for the right hemisphere; $P=0.9865$ for the left hemisphere). 

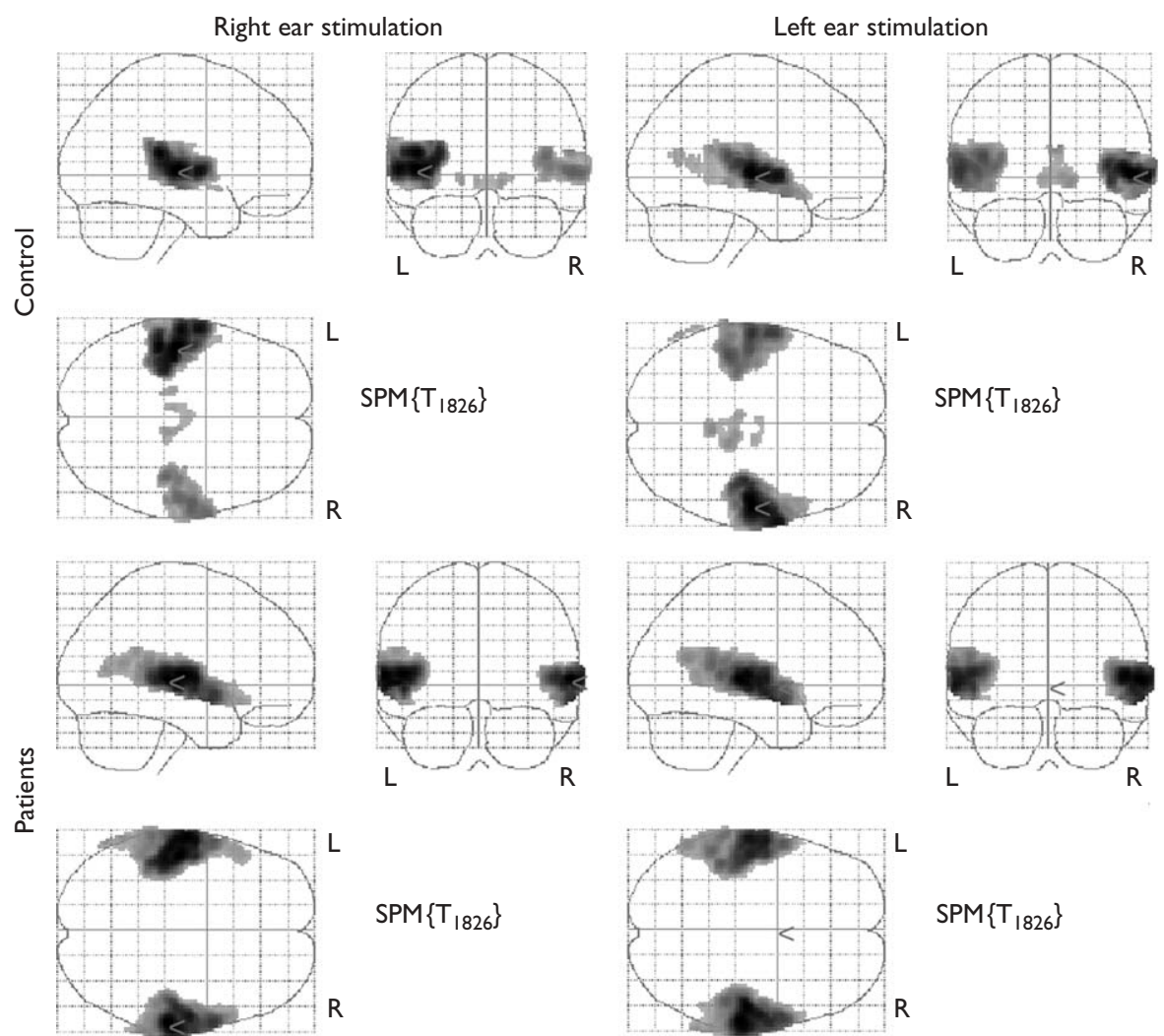

$\operatorname{SPM}\left\{T_{1826}\right\}$
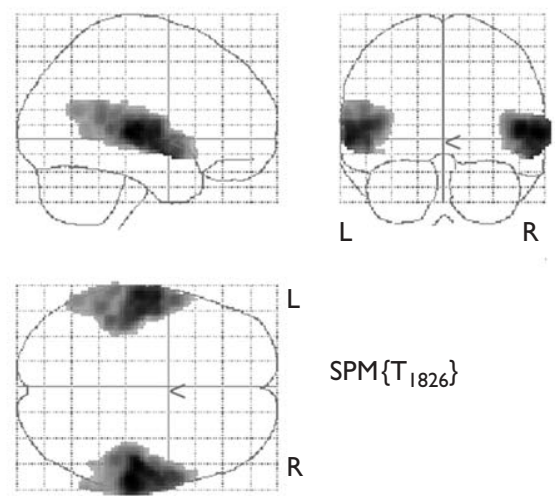

$\operatorname{SPM}\left\{\mathrm{T}_{1826}\right\}$

Fig. I Brain area activated in response to speech in normal-hearing and hearing-impaired participants. Irrespective of whether it was in the left or the right, speech signals elicited similar activation of bilateral superior temporal lobe in both groups. R, right side; L, left side. SPM, statistical parametric mapping.

Table I Group data representing regions of significant activation in normal hearing participants

\begin{tabular}{|c|c|c|c|c|c|}
\hline Anatomical locations & Brodmann area & Hemisphere & Cluster level & Voxel level & $\begin{array}{c}\text { Talairach coordinates } \\
(x, y, z) \text { for } \\
\text { local maxima }\end{array}$ \\
\hline \multicolumn{6}{|l|}{ Right ear stimulation } \\
\hline Superior temporal gyrus & 22 & Left & 3362 & 20.52 & $(-48,-19,5)$ \\
\hline Superior temporal gyrus & 29 & Left & & & $(-48,-30,14)$ \\
\hline Superior temporal gyrus & 22 & Left & & & $(-59,-15,4)$ \\
\hline Superior temporal gyrus & 22 & Right & 1307 & 11.39 & $(57,-10,2)$ \\
\hline Transverse temporal gyrus & $4 \mathrm{I}$ & Right & & & $(40,-25,12)$ \\
\hline Superior temporal gyrus & 22 & Right & & & $(63,0,0)$ \\
\hline Midbrain (red nucleus) & & Right & 155 & 7.26 & $(10,-25,-4)$ \\
\hline Midbrain (red nucleus) & & Left & & & $(-6,-2 \mathrm{l},-2)$ \\
\hline Midbrain (mammillary body) & & Left & & & $(-2,-12,-4)$ \\
\hline $\begin{array}{l}\text { Thalamus (ventral posterior lateral } \\
\text { nucleus) }\end{array}$ & & Left & 37 & 6.76 & $(-18,-21,-2)$ \\
\hline \multicolumn{6}{|l|}{ Left ear stimulation } \\
\hline Superior temporal gyrus & 22 & Right & 2829 & 27.93 & $(53,-15,3)$ \\
\hline Superior temporal gyrus & 22 & Right & & & $(63,0,0)$ \\
\hline Insula & 13 & Right & & & $(38,-21,8)$ \\
\hline Superior temporal gyrus & 22 & Left & 2888 & 19.41 & $(-59,-15,6)$ \\
\hline Superior temporal gyrus & 22 & Left & & & $(-59,-4,2)$ \\
\hline Superior temporal gyrus & 29 & Left & & & $(-44,-30,14)$ \\
\hline Thalamus & & Right & 475 & 9.60 & $(|2,-3|,-2)$ \\
\hline Posterior cingulate & 29 & Right & & & $(4,-42,19)$ \\
\hline Thalamus & & Left & & & $(-4,-25,0)$ \\
\hline Middle temporal gyrus & 19 & Left & 61 & 7.80 & $(-57,-65,16)$ \\
\hline Superior temporal gyrus & 29 & Left & & & $(-63,-52,10)$ \\
\hline Thalamus (ventral lateral nucleus) & & Right & 58 & 6.97 & $(10,-13,4)$ \\
\hline Thalamus (medial dorsal nucleus) & & Left & & & $(-4,-1 I, 4)$ \\
\hline
\end{tabular}


Table 2 Group data representing regions of significant activation in hearing-impaired patients

\begin{tabular}{|c|c|c|c|c|c|}
\hline Anatomical locations & Brodmann area & Hemisphere & Cluster level & Voxel level & $\begin{array}{c}\text { Talairach coordinates } \\
(x, y, z) \text { for } \\
\text { local maxima }\end{array}$ \\
\hline \multicolumn{6}{|l|}{ Right ear stimulation } \\
\hline Superior temporal gyrus & 22 & Left & 3896 & 23.71 & $(-61,-13,6)$ \\
\hline Superior temporal gyrus & 22 & Left & & & $(-50,-17,8)$ \\
\hline Superior temporal gyrus & 22 & Left & & & $(-55,-23,3)$ \\
\hline Superior temporal gyrus & 22 & Right & 2405 & 24.10 & $(61,-25,5)$ \\
\hline Superior temporal gyrus & 22 & Right & & & $(57,-11,4)$ \\
\hline Middle temporal gyrus & 21 & Right & & & $(61,3,-9)$ \\
\hline \multicolumn{6}{|l|}{ Left ear stimulation } \\
\hline Superior temporal gyrus & 22 & Right & 3164 & 26.75 & $(61,-23,5)$ \\
\hline Superior temporal gyrus & $4 I$ & Right & & & $(51,-19,6)$ \\
\hline Superior temporal gyrus & 22 & Right & & & $(57,-I I, 4)$ \\
\hline Superior temporal gyrus & 22 & Left & 3837 & 22.67 & $(-61,-17,1)$ \\
\hline Superior temporal gyrus & 22 & Left & & & $(-59,-8,4)$ \\
\hline Transverse temporal gyrus & 41 & Left & & & $(-51,-17,8)$ \\
\hline
\end{tabular}

\section{Discussion}

This blocked-design fMRI study demonstrates similarity in brain activation patterns during speech perception between congenital hearing-impaired and normal-hearing participants. Our results support the hypothesis that the functional differentiation in areas of the brain used for auditory processing is not affected greatly in patients with bilateral hearing impairment from childhood, if enough acoustic stimulation had been obtained, even in patients with profound hearing loss [6].

Hearing loss might raise excitability in the auditory cortex, although developmental hearing impairment compromises sound discrimination, speech acquisition, and cognitive function. Kotak et al. [7] exhibited significantly larger thalamocortically and intracortically evoked excitatory synaptic responses in developing gerbils with sensorineural hearing loss. It remains unclear, however, whether hearing loss increases excitability in the auditory cortex of humans. In our study, the signal intensity and extent of activation seemed to be increased slightly in hearingimpaired patients, as shown in Fig. 1. It is hard to explain these findings, but it is possible that ipsilateral inhibition or increased excitability in the subcortical auditory nuclei and the auditory cortex deteriorated in response to monaural stimulation in our patients.

Connectivity analyses have shown that most auditory information crosses to the contralateral side in the lower brainstem and is then channeled ipsilaterally towards the auditory cortex. By fMRI study, the lateralization ratios between left and right hemispheric response areas were 3.4-5.2 for monaural stimulation and nearly balanced for binaural stimulation in normal-hearing patients in response to passively heard $1000 \mathrm{~Hz}$ pulsed sine tones [8]. Increasing the complexity of the monaurally presented sound stimulus seems to decrease the likelihood of a contralaterally asymmetric response. In addition, various listening tasks might affect contralaterality. For example, strong contralaterality effects during monaural stimulation were noted only in the posterior STG, but not in the whole STG, during tone-identification tasks [9]. In this study, except during right ear stimulation in normal-hearing patients, we failed to demonstrate significant contralateral hemispheric dominance for speech sounds, possibly because our experiment required only passive listening but not linguistically demanding tasks.

\section{Conclusion}

As long as peripheral acoustic stimulation has not been totally absent from childhood, the classical activation pattern can be elicited in patients with congenital bilateral, moderate-to-severe, hearing impairment.

\section{References}

1. Emmorey K, Allen JS, Bruss J, Schenker N, Damasio H. A morphometric analysis of auditory brain regions in congenitally deaf adults. Proc Nat Acad Sci 2000; 100:10049-10054.

2. Lee DS, Lee JS, Oh SH, Kim SK, Kim JW, Chung JK, Lee MC. Cross-modal plasticity and cochlear implant. Nature 2001; 409:149-150.

3. Lee JS, Lee DS, Oh SH, Kim CS, Kim JW, Hwang CH, et al. PET evidence of neuroplasticity in adult auditory cortex of postlingual deafness. J Nucl Med 2003; 44:1435-1439.

4. Welzl-Muller K, Stephan K. Speech recognition performance with hearingaid in noiseless and noisy conditions. Audiol Akustik 1988; 27:108-119.

5. Hall DA, Summerfield AQ, Goncalves MS, Foster JR, Palmer AR, Bowtell RW. Time-course of the auditory BOLD response to scanner noise. Magn Reson Med 2000; 43:601-606.

6. Hirano S, Naito Y, Kojima H, Honjo I, Inoue M, Shoji K, et al. Functional differentiation of the auditory association area in prelingually deaf subjects. Auris Nasus Larynx 2000; 27:303-310.

7. Kotak VC, Fujisawa S, Lee FA, Karthikeyan O, Aoki C, Sanes DH. Hearing loss raises excitability in the auditory cortex. J Neurosci 2005; 25:3908-3918.

8. Scheffler K, Bilecen D, Schmid N, Tschopp K, Seelig J. Auditory cortical responses in hearing subjects and unilateral deaf patients as detected by functional magnetic resonance imaging. Cereb Cortex 1998; 8:156-163.

9. Jancke L, Wustenberg T, Schulze K, Heinze HJ. Asymmetric hemodynamic responses of the human auditory cortex to monaural and binaural stimulation. Hear Res 2002; 170:166-178. 